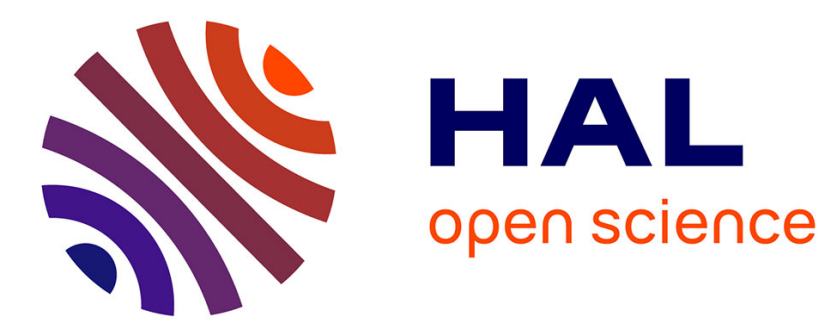

\title{
Photoacoustic transformation in magnetically active media under the interaction of opposing light waves
}

\author{
G. Mityurich, V. Zelenyi, A. Zelenyi, A. Serdyukov
}

\section{To cite this version:}

G. Mityurich, V. Zelenyi, A. Zelenyi, A. Serdyukov. Photoacoustic transformation in magnetically active media under the interaction of opposing light waves. Journal de Physique IV Proceedings, 1994, 04 (C7), pp.C7-769-C7-772. 10.1051/jp4:19947181 . jpa-00253243

\section{HAL Id: jpa-00253243 https://hal.science/jpa-00253243}

Submitted on 1 Jan 1994

HAL is a multi-disciplinary open access archive for the deposit and dissemination of scientific research documents, whether they are published or not. The documents may come from teaching and research institutions in France or abroad, or from public or private research centers.
L'archive ouverte pluridisciplinaire HAL, est destinée au dépôt et à la diffusion de documents scientifiques de niveau recherche, publiés ou non, émanant des établissements d'enseignement et de recherche français ou étrangers, des laboratoires publics ou privés. 


\title{
Photoacoustic transformation in magnetically active media under the interaction of opposing light waves
}

\author{
G.S. Mityurich, V.P. Zelenyi, A.P. Zelenyi* and A.N. Serdyukov \\ Gomel State University, Soviet St. 104, Gomel 246699, Belarus \\ * Moscow Institute of Physics and Technology, Dolgoprudnyi, 141700 Moscow Region, Russia
}

\begin{abstract}
The process of thermo-optical excitation of sound by opposing light waves is studied in the magnetically active medium. The influence of natural and induced optical activity on the resulting photoacoustic (PA) response is analyzed. A method of controlling the amplitude of PA signal is proposed. The method is based on the possibility to affect the interference energy dissipation by an external magnetic field.
\end{abstract}

\section{INTRODUCTION}

PA methods can increase the accuracy in measuring the small parameters which are responsible for absorption and dichroism for a number of naturally gyrotropic and magnetically active samples. Investigations directed to the effectiveness increase of the thermo-optical transformation and to the informative growth of photoacoustical spectroscopy do not lose their present-day significance.

The process of interaction of electromagnetic waves in absorbing materials is accompanied by the display of a series of specific effects which can be applied in the PA technique. For example, in [1] a method of ultrasonic wave excitation in absorbing condensed media is considered. The method is based on impulse heating by interference field of coherent light excitation and allows measurement of velocity and sound damping in samples. The authors [2] examined experimentally a phenomenon of tunnel-effect electromagnetic interference in $\mathrm{Ti}$ metal films at interaction of two contrary light beams when it was shown that the value of the transmission coefficient essentially depends on the opposing light beams intensity, phase difference, polarization and sample dissipative properties. Studies of similar phenomenon in absorbing gyrotropic media has lead to a series of characteristic peculiarities connected with display of thin optical effects, i. e., circular birefringence and circular dichroism.

\section{THEORETICAL MODEL}

This work is devoted to the investigation of thermo-optical excitation of sound in a magnetically active medium under the interaction of opposing light waves.

It is found from the Maxwell equations for plane electromagnetic waves and from the 
material equations for the gyrotropic medium in an external magnetic field [3]

$$
\begin{aligned}
& \mathbf{E}=\left(1 / \varepsilon_{0}+i \mathbf{G}^{\times}\right) \mathbf{D}+i \sigma_{0} \mathbf{B} \\
& \mathbf{H}=\mathbf{B}-i \sigma_{0} \mathbf{D}
\end{aligned}
$$

that the amplitudes of the light fields in the medium and the volumetric energy dissipation rate of the opposing light waves can be determined as follows

$$
\begin{aligned}
Q^{ \pm} & =Q^{ \pm}+Q_{\mathrm{int}}^{ \pm} \\
Q^{ \pm} & =\frac{\omega}{4 \pi}\left(\frac{\varepsilon^{\prime \prime}}{\varepsilon^{\prime 2}+\varepsilon^{\prime \prime 2}} \pm G^{\prime \prime}\right)\left(A_{ \pm} \exp \left\{-\alpha_{ \pm} x\right\}+B_{ \pm} \exp \left\{\alpha_{\mp}^{\prime} x\right\}\right) \\
Q_{\mathrm{int}}^{ \pm} & =\frac{\omega}{4 \pi}\left(\frac{\varepsilon^{\prime \prime}}{\varepsilon^{\prime 2}+\varepsilon^{\prime \prime 2}} \pm G^{\prime \prime}\right)\left(C^{ \pm} \exp \left\{2 i \frac{\pi}{\lambda} x\left(n_{ \pm}+n_{\mp}^{\prime *}\right)\right\}+\mathrm{c} . \mathrm{c} .\right) \\
C^{ \pm} & =R^{ \pm}+T^{ \pm}, \quad R^{ \pm}=X^{ \pm} e^{i\left(\varphi_{0}-\varphi\right)}+Y^{ \pm} e^{-i\left(\varphi_{0}-\varphi\right)} \\
T^{ \pm} & =E_{0}^{ \pm 2} G_{0}^{(1,2)} G_{1}^{(2,1) *}+E^{\mp 2} R_{1}^{(1,2)} R_{0}^{(2,1) *} \\
\alpha_{ \pm} & =\frac{4 \pi}{\lambda} \operatorname{Im}\left[n_{ \pm}\right] \quad \alpha_{ \pm}^{\prime}=\frac{4 \pi}{\lambda} \operatorname{Im}\left[n_{ \pm}^{\prime}\right] \\
n_{ \pm} & =\left(\sqrt{1 / \varepsilon_{0} \pm G} \pm \sigma_{0}\right)^{-1 / 2}, \quad n_{ \pm}^{\prime}=\left(\sqrt{1 / \varepsilon_{0} \mp G} \pm \sigma_{0}\right)^{-1 / 2}
\end{aligned}
$$

The term $Q_{\text {int }}^{ \pm}$is connected with the interference flow in the medium. In equation (1) and (2), $\varepsilon_{0}=\varepsilon^{\prime}+i \varepsilon^{\prime \prime}$ is the tensor of the dielectric permeability, $\sigma_{0}=\sigma_{0}^{\prime}+\sigma_{0}^{\prime \prime}$ is the pseudoscalar parameter of natural optical activity, and $G=G^{\prime}+i G^{\prime \prime}$ is the nonsymmetrical tensor of rank two which is dual to the magnetic gyration vector $\mathbf{G}$. The terms $\sigma_{0}^{\prime}$ and $G^{\prime}$ describe the natural and induced specific rotation of the polarization plane of the electromagnetic waves, $\sigma_{0}^{\prime \prime}$ and $G^{\prime \prime}$ represent natural and magnetic circular dichroism. The rest of the notations in (2) correspond to those adopted in [4].

The PA signal in the gas microphone cell is calculated from equation (2) in the assumption of the Rosencwaig-Gersho model. The complex amplitude of temperature field on the boundary of $x=d / 2$ can be found as follows

$$
\begin{aligned}
& \theta^{ \pm}=\frac{2}{\Delta}\left(\Theta_{0}^{ \pm} \operatorname{sh}\left(\sigma_{s} d\right)+\Theta_{1}^{ \pm} \operatorname{ch}\left(\sigma_{s} d\right)-s_{0} F_{3}^{ \pm}-F_{4}^{ \pm}-Q_{2}^{ \pm}\right), \\
& \Theta_{0}^{ \pm}=F_{3}^{ \pm}-s_{0}\left(F_{4}^{ \pm}+Q_{1}^{ \pm}\right), \quad \Theta_{1}^{ \pm}=s_{0} F_{3}^{ \pm}-F_{4}^{ \pm}-Q_{2}^{ \pm} .
\end{aligned}
$$

In equation (3), $Q_{1}^{ \pm}$and $Q_{2}^{ \pm}$are the interference terms of $Q_{\text {int }}^{ \pm}$energy dissipation on the $x=-d / 2$ and $x=d / 2$ boundaries. The notations in (3) correspond to those adopted in [4].

\section{ANALYSIS}

In view of the awkwardness of (2), (3) a numerical analysis of the received relations was carried out. If the sample thickness $d$ is greater than $1 \mathrm{~mm}$, the amplitude of PA signal is strongly dependent on the real part of the magnetic gyration parameter (Fig. 1), and it can grow by several orders of magnitude, depending on the characteristic parameters (direction of the external magnetic field, initial phase difference (Fig. 1), polarization, and modulation frequency of the light beams). Maximum amplitude rise of PA signal similar to [4] is displayed under the interaction of two linear polarized in one plane electromagnetic waves with the initial phase difference $\Delta \varphi=\pi / 2$, as well as light beams with right circular polarization with 
this phenomena taking place only at the contrary interaction of light waves. Poor dependence of PA signal amplitude and phase on $G^{\prime}$ at $d \leq 1 \mathrm{~mm}$ is obviously defined by the value of polarization plane rotation of electromagnetic waves in crystal which is directly proportional to the light path in the sample. PA signal amplitude change may be experimentally applicable in the transmitted PA sell [5] being put into external to the light path in the sample. Interference flow is absent $\left(Q_{\text {int }}^{ \pm}=0\right)$ when the electric field strength of one of the beams is equal zero. PA signal amplitude in this case depends on the real part of magnetic gyration parameter only in the domain of small thickness $d<1 \mathrm{~mm}$. Variation $G^{\prime}$ however does not result in significant growth of a resulting signal value. This is connected with the redistribution of energy between incident and reflected light waves in a sample. Moreover the influence of reflection decreases with the growth of the crystal thickness. One can control the amplitude and phase characteristics value of the PA signal by varying the initial phases of the interacting light beams. If natural $\left(\sigma^{\prime} \neq 0, \sigma^{\prime \prime} \neq 0\right)$ and magnetic activities $\left(G^{\prime} \neq 0, G^{\prime \prime} \neq 0\right)$ take place, a significant change of the amplitude of the PA response can be found in the crystal sample (Fig.2).

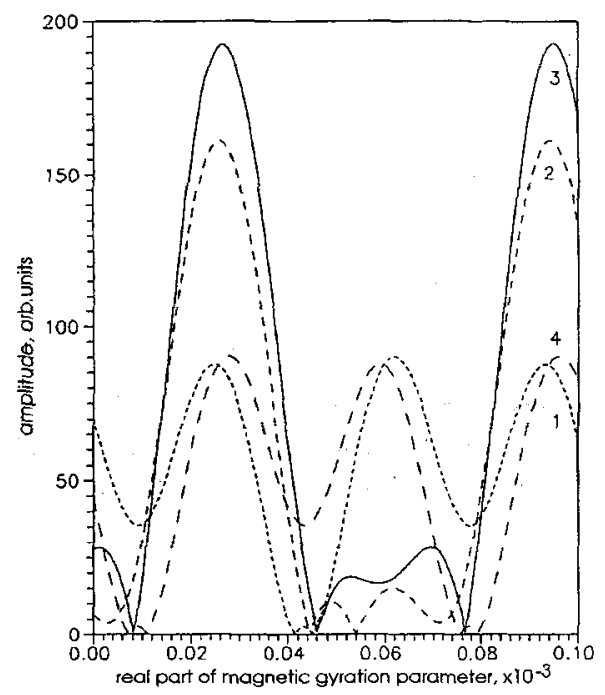

Figure 1. Dependence of PA signal amplitude on the real part of the magnetic gyration parameter at different values of the initial phase difference of the light beams $(\Delta \varphi, \mathrm{rad}):. 1-0,2-\pi / 4,3-\pi / 2,4-$ $\pi$.

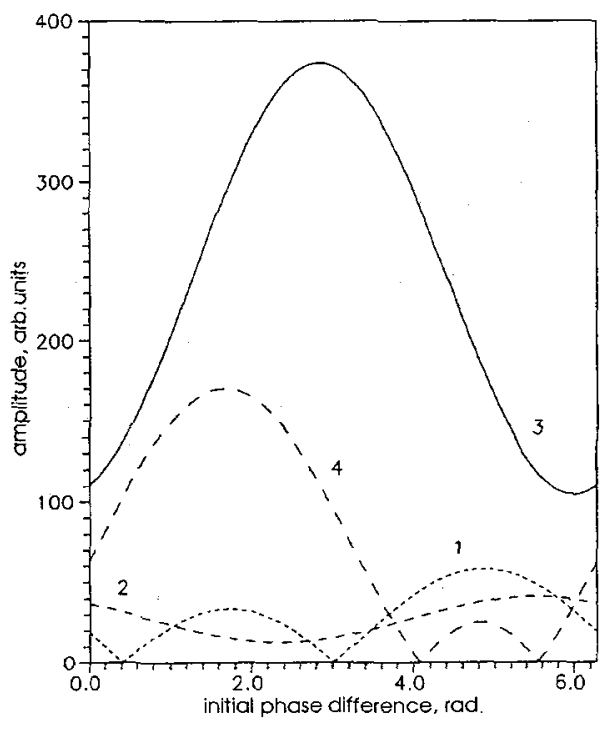

Figure 2. Dependence of PA signal amplitude on the initial phase difference of the light beams at different values of the real part of the magnetic gyration parameter: $1-\sigma^{\prime}=\sigma^{\prime \prime}=G^{\prime}=G^{\prime \prime}=0 ; 2-\sigma^{\prime}=$ 1. $\times 10^{-4}, \sigma^{\prime \prime}=1 . \times 10^{-5}, G^{\prime}=G^{\prime \prime}=0 ; 3$ $-G^{\prime}=1 . \times 10^{-4}, G^{\prime \prime}=1 . \times 10^{-5}, \sigma^{\prime}=$ $\sigma^{\prime \prime}=0,4-\sigma^{\prime}=1 . \times 10^{-4}, \sigma^{\prime \prime}=1 . \times$ $10^{-5}, G^{\prime}=1 . \times 10^{-4}, G^{\prime \prime}=1 . \times 10^{-5}$. 
Variations of the circular magnetic dichroism parameter also change the PA response, the PA signal being maximal when the condition $G^{\prime \prime} \approx \varepsilon^{\prime \prime} /\left(\varepsilon^{\prime 2}+\varepsilon^{\prime 2}\right)$ from (2) is true. The growth of the PA signal amplitude, which is determined by the temperature of the surface of the magnetically active sample, is due to the redistribution of the absorbed heat over the sample.

\section{CONCLUSION}

Thus, the gyrotropic characteristics of the examined sample affect the PA signal forming mechanism. It was found that under the opposite interaction of light waves in magnetically active media the change of $G^{t}$ parameter, which is responsible for specific rotation of a light polarization plane, leads to increasing of PA signal amplitude by several orders (see Fig.1) under the constant intensity of the opposite light beams. By placing the sample in the PA cell, which is in an external magnetic field and changing its value, one can affect the interference redistribution of energy dissipation. This lets us propose a method of controlling amplitude and phase characteristics of a PA signal.

\section{REFERENCES}

[1 ] E. V. Ivakin, A .M. Lazaruk, I. P. Petrovich, and A. S. Rubanov, Kvantovaya Elektronika, (1977) 4, 2421-2426.

[2 ] V. V. Sidorenkov and V. V. Tolmachev, Pis'ma v Zh. Tekh. Fiz. (1989) 15, 34-37.

[3 ] N. N. Khilo and A. N. Serdyukov, Zh. Prikl. Spektrosk. (1976) 25, 169-173.

[4] G. S. Mityurich, V. P. Zelenyi, and A. N. Serdyukov, Photoacoustic transformation in gyrotropic media at interaction of two light beams, in Acousto-Optics and Applications, A. Śliviński, P. Kwiek, B. Linde, and A. Markiewicz, Editors, Proc. SPIE 1844, (1992) pp. 309-318.

[5 ] Cahen D. Rev. Sci. Instrum. (1981) 52, 1306-1310. 